\title{
A Forbidden Transition Allowed for Stars
}

\section{The discovery of an exceptionally strong "forbidden" beta-decay involving fluorine and neon could change our understanding of the fate of intermediate-mass stars.}

\section{by Carla Frohlich*}

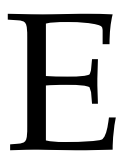
very year roughly 100 billion stars are born and just as many die. To understand the life cycle of a star, nuclear physicists and astrophysicists collaborate to unravel the physical processes that take place in the star's interior. Their aim is to determine how the star responds to these processes and from that response predict the star's final fate. Intermediate-mass stars, whose masses

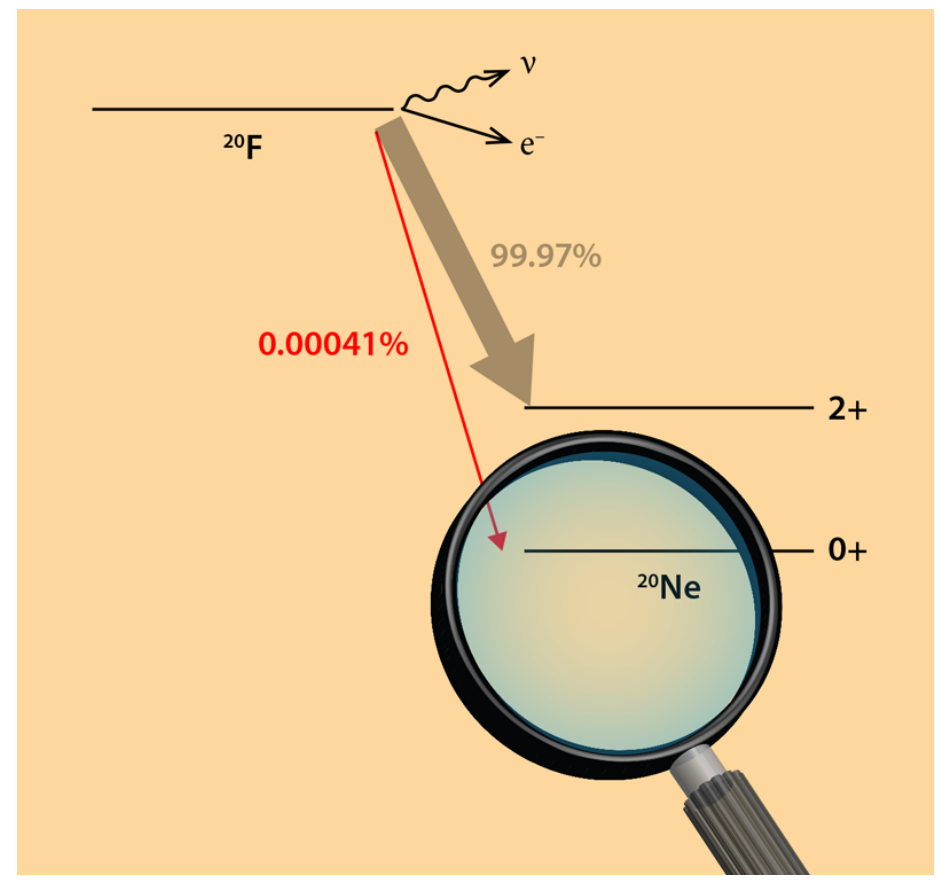

Figure 1: Researchers have measured the forbidden nuclear transition between ${ }^{20} \mathrm{~F}$ and ${ }^{20} \mathrm{Ne}$. This measurement allowed them to make a new calculation of the electron-capture rate of ${ }^{20} \mathrm{Ne}$, a rate that is important for predicting the evolution of intermediate-mass stars. (APS/Alan Stonebraker)

*Department of Physics, North Carolina State University, Raleigh, NC, USA lie somewhere between 7 and 11 times that of our Sun, are thought to die via one of two very different routes: thermonuclear explosion or gravitational collapse. Which one happens depends on the conditions within the star when oxygen nuclei begin to fuse, triggering the star's demise. Researchers have now, for the first time, measured a rare nuclear decay of fluorine to neon that is key to understanding the fate of these "in between" stars [1, 2]. Their calculations indicate that thermonuclear explosion and not gravitational collapse is the more likely expiration route.

The evolution and fate of a star strongly depend on its mass at birth. Low-mass stars-such as the Sun-transition first into red giants and then into white dwarfs made of carbon and oxygen as they shed their outer layers. Massive stars-those whose mass is at least 11 times greater than the Sun's-also transition to red giants, but in the cores of these giants, nuclear fusion continues until the core has turned completely to iron. Once that happens, the star stops generating energy and starts collapsing under the force of gravity. The star's core then compresses into a neutron star, while its outer layers are ejected in a supernova explosion. The evolution of intermediate-mass stars is less clear. Predictions indicate that they can explode both via the gravitational collapse mechanism of massive stars and by a thermonuclear process [3-6]. The key to finding out which happens lies in the properties of an isotope of neon and its ability to capture electrons.

The story of fluorine and neon is tied to what is known as a forbidden nuclear transition. Nuclei, like atoms, have distinct energy levels and thus can exist in different energy states. For a given radioactive nucleus, the conditions within a star, such as the temperature and density of its plasma, dictate its likely energy state. The quantum-mechanical properties of each energy state then determine the nucleus' likely decay path. The decay is called allowed if, on Earth, the decay path has a high likelihood of occurring. If, instead, the likelihood is low, the transition is termed forbidden. But in the extreme conditions of a star's interior, these forbidden transitions can occur much more frequently. Thus, when researchers measure a nuclear reaction in the laboratory, the very small contribution from a forbidden transition is often the most critical one to measure for the astrophysics applica- 
tions.

One important forbidden transition for stars connects fluorine $(\mathrm{F})$ and neon $(\mathrm{Ne})$, either via the decay of ${ }^{20} \mathrm{~F}$ to ${ }^{20} \mathrm{Ne}$, or via the capture of an electron by ${ }^{20} \mathrm{Ne}$ to produce ${ }^{20} \mathrm{~F}$. Knowing one of these processes you can determine the other. Most often, the transition involves the excited ${ }^{20} \mathrm{Ne}$ nucleus $\left({ }^{20} \mathrm{Ne}^{2+}\right)$, but under certain conditions-which are speculated to exist in intermediate-mass stars-the transition instead proceeds predominantly via ${ }^{20} \mathrm{Ne}$ in its ground state $\left({ }^{20} \mathrm{Ne}^{0+}\right)$. The explosion mechanism of a star is predicted to depend critically on the rate of electron capture on ${ }^{20} \mathrm{Ne}$, so measuring how frequently it proceeds through ${ }^{20} \mathrm{Ne}^{0+}$ is key to understanding a star's fate. It was this transition between ${ }^{20} \mathrm{~F}$ and ${ }^{20} \mathrm{Ne}^{0+}$ that Oliver Kirsebom, now at Dalhousie University in Canada, and his colleagues set out to measure.

In their experiments, which were carried out at JYFL Accelerator Laboratory in Finland, Kirsebom and colleagues bombarded a carbon foil with a beam of ${ }^{20} \mathrm{~F}$ nuclei, implanting the foil with the radioactive nuclei [2]. They then monitored the radioactive decay of the fluorine nuclei, a process that emits an energetic electron and a neutrino. They focused the energetic electrons produced in the decay onto a scintillator detector, measuring their energies from the light produced when the electrons hit.

The team measured electrons with energies above 5.8 $\mathrm{MeV}$. These electrons can only have arisen from the forbidden transition of ${ }^{20} \mathrm{~F}$ to ${ }^{20} \mathrm{Ne}^{0+}$. When ${ }^{20} \mathrm{~F}$ decays to ${ }^{20} \mathrm{Ne}^{2+}$, $1.634 \mathrm{MeV}$ of the energy released by the decay, the so-called decay energy, is lost by the photon that is subsequently emitted by the excited Ne. But when ${ }^{20} \mathrm{~F}$ decays to ${ }^{20} \mathrm{Ne}^{0+}$ the entire decay energy $(7.024 \mathrm{MeV})$ transfers to the electron and the antineutrino. Because of this difference, electrons emitted by this forbidden transition carry more energy than those emitted by the more common transition. By carefully counting the number of electrons of each energy, the team determined that $0.00041 \%$ - or roughly 1 in 250,000 - of the decays of ${ }^{20} \mathrm{~F}$ lead to ${ }^{20} \mathrm{Ne}^{0+}$. Albeit tiny, this percentage is surprisingly large and makes it the second strongest forbidden transition measured in any nucleus.

To understand the impact that their result has on the death of intermediate-mass stars, Kirsebom and his colleagues used their decay likelihood measurement to calculate the electron-capture rate for ${ }^{20} \mathrm{Ne}$ in the stellar environment [1]. They found an electron-capture rate 8 orders of magnitude larger than that predicted by previous calculations. Inputting this larger capture rate into simulations of intermediate-mass stars, they observed earlier heating of the stellar core and fusing of oxygen at lower densities. Because of these observations nuclear fusion was found to be less energetic when compared with predictions made using previous, smaller electron-capture rates. In all their simulations, the team observed that the stars expired by thermonuclear explosion. This explosion only partially disrupted the star, leaving behind a white dwarf that consisted mostly of oxygen, neon, and magnesium.

The results obtained by Kirsebom and his colleagues are a milestone in precision nuclear astrophysics. It took a dedicated experimental setup-and several decades since the first attempt- to measure this forbidden beta-decay transition, which was the last remaining nuclear physics uncertainty in the evolution of intermediate stellar cores. But the evolution of these stars still has open questions. Researchers now need to focus on thermodynamics and understanding whether these stellar cores can become unstable because of convection, which mixes the material and transport energy from the core outwards. This mixing could counteract the effects of the enhanced electron-capture rate, which would mean that the star would instead expire by gravitational collapse. Only when we understand the details of all the processes occurring within stars can we unlock the secrets of how these astrophysical objects evolve and die.

This research is published in Physical Review Letters and Physical Review C.

\section{REFERENCES}

[1] O. S. Kirsebom et al., "Discovery of an exceptionally strong $\beta$-decay transition of ${ }^{20} \mathrm{~F}$ and implications for the fate of intermediate-mass stars," Phys. Rev. Lett. 123, 262701 (2019).

[2] O. S. Kirsebom et al., "Measurement of the $2^{+} \rightarrow 0^{+}$groundstate transition in the $\beta$ decay of ${ }^{20} \mathrm{~F}$," Phys. Rev. C 100, 065805 (2019).

[3] H.-Th. Janka, B. Müller, F. S. Kitaura, and R. Buras, "Dynamics of shock propagation and nucleosynthesis conditions in O-NeMg core supernovae," Astron. Astrophys. 485, 199 (2008).

[4] S. Jones et al., "Do electron-capture supernovae make neutron stars?" Astron. Astrophys. 593, A72 (2016).

[5] D. Radice, A. Burrows, D. Vartanyan, M. A. Skinner, and J. C. Dolence, "Electron-capture and Low-mass Iron-core-collapse Supernovae: New Neutrino-radiation-hydrodynamics Simulations," Astrophys. J. 850, 43 (2017).

[6] S. Zha, S.-C. Leung, T. Suzuki, and K. Nomoto, "Evolution of ONeMg Core in Super-AGB Stars toward Electron-capture Supernovae: Effects of Updated Electron-capture Rate," Astrophys. J. 886, 22 (2019).

10.1103/Physics.12.151 